\title{
Technology Advancement in Vehicle Monitoring System for Accident Prevention
}

\author{
N.P. Chai, W.A.W.Z. Abidin, W. H. W. Ibrahim and K. Hong Ping
}

\begin{abstract}
Significant development in urbanized cities causes vehicle ownership to grow with rapid rate. As a result, the saturation of vehicle in dense cities leads to insufficient transportation infrastructure, which causes an increase in road accident occurrences. The condition of vehicle and driver such as poor engine operation system, speeding, reckless driving, drowsy driving, drunken driving are the factors of accidents. Vehicle monitoring system is thus very important to be deployed as an effort for accident prevention to reduce the number of road accidents. This paper reviews the recent progress and development of technologies in vehicle monitoring system for accident prevention. Various technologies in the system include Global Positioning System (GPS), Geographical Information System (GIS), Global System for Mobile (GSM), General Packet Radio Service (GPRS), image processing, Fuzzy Logic, data fusion and so forth. The issues and challenges in vehicle monitoring system are also presented in this paper.
\end{abstract}

Keywords: Vehicle Monitoring System, accident prevention, technologies, issues and challenges

\section{INTRODUCTION}

I $\mathrm{N}$ most of the developing countries, drastic increase of vehicle ownership generates a great demand on transportation infrastructures. However, the road and rail network construction could hardly fulfill the requirement of ever-increasing traffic demands and thus worsen traffic environment and road safety issues [1].

The world car registration is found increased by $29 \%$ since 2000 to 2010 and the countries with five highest average annual percentage change of car registration are found to be developing countries, namely China, Indonesia, South Korea, India, and Malaysia [2]. Within the timeline, China had contributed the most to car production with a dramatic increase in percentage of $1470 \%$ [2].

Since year 2000, Beijing with an average annual growth rate of 3.64\% of urban road length could not support the number of vehicle with average annual growth rate of $10.91 \%$ [3]. Insufficient transportation infrastructure with excess vehicles will bring adverse impacts such as the increase in road accidents. With the implementation of Intelligent Transportation System (ITS), the road capacity can be improved and the number of traffic accidents can be reduced.

The Malaysian Institute of Road Safety Research (MIROS) statistic showed that the number of registered vehicles and road accidents in Malaysia have been increasing since year 2007 onwards [4]. Road accidents cause deaths, casualties and economy losses, affecting Gross Domestic Product (GDP) of a country. In order to prevent road accidents, vehicle monitoring system as a kind of ITS is suggested to be developed.

Vehicle monitoring system is an intelligent system that integrates hardware and software and utilizes surveillance, communication and information technologies to observe the condition or situation of a vehicle by the user, and third party over the time. The system can provide warning to the driver if any possible dangerous or hazardous condition is detected while the third party can observe and alert the driver when any driver abnormal condition is discovered.

Normally, the vehicle monitoring system consists of vehicle terminal, communication link and monitoring centre. The system collects various data at vehicle terminal in real time and then transmits the data via communication link to the monitoring centre for surveillance purposes. The general block diagram of vehicle monitoring system is shown in Figure 1.

N.P. Chai is with the Department of Electronic Engineering, Faculty of Engineering, Universiti Malaysia Sarawak (UNIMAS), 94300 Kota Samarahan, Sarawak, Malaysia (phone: +60 168649206; fax: +6082583410; e-mail: chaineeping@ gmail.com)

W.A.W.Z. Abidin is with the Department of Electronic Engineering, Faculty of Engineering, Universiti Malaysia Sarawak (UNIMAS), 94300 Kota Samarahan, Sarawak, Malaysia (e-mail: wzaazlan@feng.unimas.my).

W. H. W. Ibrahim is with the Department of Civil Engineering, Faculty of Engineering, Universiti Malaysia Sarawak (UNIMAS), 94300 Kota Samarahan, Sarawak, Malaysia (e-mail: wiwhashim@feng.unimas.my).

K. Hong Ping is with the Department of Electronic Engineering, Faculty of Engineering, Universiti Malaysia Sarawak (UNIMAS), 94300 Kota Samarahan, Sarawak, Malaysia (e-mail: hpkismet@feng.unimas.my). 


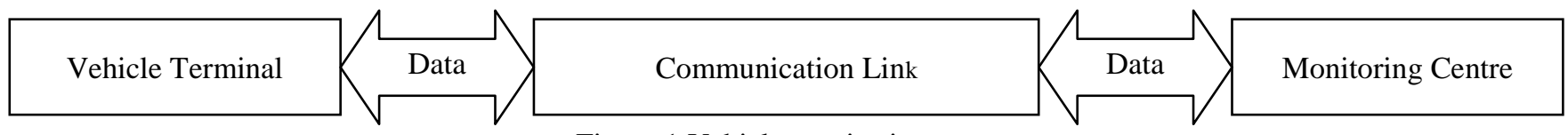

Figure 1 Vehicle monitoring system

For vehicle terminal, the positioning technology is necessary in order to identify the location of vehicle in real time. The positioning technologies are divided into three categories, which are satellite-based positioning, network-based positioning and local positioning [5]. The satellite-based positioning has high accuracy and commonly utilized in vehicle monitoring systems. Network-based positioning is rarely utilized in vehicle monitoring system due to its low accuracy compared to satellite-based positioning. Local positioning is not suitable for vehicle monitoring purposes since it only provides location information within a restricted area based on the short-range signal transmission.

Satellite-based positioning locates the satellite receiver through mathematical calculations involving the orbit and time information from a number of satellites of Global Navigation Satellite System (GNSS). The United State of America GPS and Russian GLONASS are fully operational GNSS while European Union Galileo and China Compass are GNSS in the process of development [6]-[8]. In addition to the GNSS, there are regional satellite systems such as India Regional Navigation Satellite System (IRNSS) and Japan Quasi-Zenith Satellite System (QZSS) [8]. There are also different kinds of satellite-based methods and system extensions that can improve the accuracy and reliability of GNSS such as Assisted-GPS (AGPS) and Differential-GPS (DGPS) positioning.

As GPS and GLONASS are the fully operational GNSS, the evaluated attributions of these two GNSS will be compared. From Table 1, GPS can be seen as having slightly lower accuracy compared to GLONASS. However, GPS has the advantage of being the most mature and practical technology that is now extensively used in navigation, positioning or vehicle monitoring system [9]-[10].

Table 1 Comparison between GPS and GLONASS [9]-[11]

\begin{tabular}{|c|c|c|}
\hline Evaluated Attribute & GPS & GLONASS \\
\hline Accuracy & $7 \mathrm{~m}$ & $6 \mathrm{~m}$ \\
\hline Application in VMS & High & Low \\
\hline
\end{tabular}

Apart from positioning technology, sensor system with essential data fusion technique is required to detect the condition of vehicle and driver at vehicle terminal in real-time. A variety of sensors are integrated into the system to obtain information related to safety in order to reduce the possibility of accident occurrence while data fusion is a technique of collection to combine data from multiple sensors [12].

Wireless Communication link is a critical element as it is the transmission medium that links between the vehicle terminal and monitoring centre. Communication link can be wired or wireless. The common wired media are twisted pair cable, coaxial cable and optical fiber. However, research on communication in vehicle monitoring center emphasizes on wireless communication link due to its mobility and impressive transmission range.

The wireless communication technologies include Bluetooth, Wireless Local Area Network (WLAN), Global System for Mobile Communications (GSM) and General Packet Radio Signal (GPRS) [13]-[14]. Bluetooth is short-range wireless communication technologies with transmission range limited to 15-100m [15], which is only suitable for restricted area activities but not for vehicle monitoring. WLAN is suitable for long-range transmission but it is not as common as GSM and GPRS [15]-[22], in vehicle monitoring. This is because WLAN technology is not available in some rural areas as GSM network does.

GSM is a $2.0 \mathrm{G}$ technology while GPRS which extends the GSM packet data transfer capability is a $2.5 \mathrm{G}$ technology. GSM is using circuit-switched network with data transfer speed of $9.6 \mathrm{~kb} / \mathrm{s}$. In contrast, GPRS is using packet-switched network with data transfer speed of $53.6-115 \mathrm{~kb} / \mathrm{s}$, which is faster than GSM. Both of the GSM and GPRS use $850 / 900 / 1800 / 1900 \mathrm{Mhz}$ as transmission frequency and both are reliable global communication networks which allows data transmission between two mobile phones regardless of time and place as long as there are base stations nearby. They presented high mobility, wide coverage, wide range, low cost, high efficiency, and easy merge with other technologies [15]. Another added value is that no investment is needed on its infrastructure. The comparisons between GSM and GPRS are shown in Table 3.

Table 2 Comparison between GSM and GPRS [15], [23]-[24]

\begin{tabular}{|c|c|}
\hline GSM & GPRS \\
\hline $2.0 \mathrm{G}$ & $2.5 \mathrm{G}$ \\
\hline Circuit-Switched & Packet-Switched \\
\hline $9.6 \mathrm{~kb} / \mathrm{s}$ & $53.6-115 \mathrm{~kb} / \mathrm{s}$ \\
\hline
\end{tabular}


At the monitoring centre, Geographical Information System (GIS) is used to store, analyze and display geographicallyreferenced information [25]. Geographical information can be categorized into spatial data and attribute data [26]. Spatial data is used to visualize and manipulate the real world objects such as roads, buildings and crime locations in a computer model. The most commonly defined spatial elements are points, lines and areas. Attribute data describes the real world objects in terms of text such as the name and width of a road.

These two components are stored in different databases but both data are necessary to be utilized in the commercial positioning system. GIS technology is deployed in most of the vehicle tracking system as they are dependent to large amount of geographical spatial information. MapX, Google Map and Google Earth are the common used GIS.

\section{VEHICLE MONITORING SYSTEM}

\section{A. Vehicle Terminal}

A lot of vehicle monitoring systems have been proposed and implemented in the past few years [9]-[10], [15]-[22]. Research works on vehicle monitoring systems are important for the purpose of accident prevention. Various technologies are integrated into the systems to improve the performance.

The vehicle monitoring system can be categorized as in-vehicle and on-road surveillance system. In-vehicle surveillance system embeds the electronic device or onboard equipment on the vehicle and most likely requires wireless communication as transmission medium. On the other hand, on-road surveillance system installs the electronic devices or monitoring station at the roadside and may deploy wired or wireless communication technologies.

GPS has been the most popular technology used in vehicle monitoring system [9]-[10], [19]-[22]. The GPS receiver is installed at the vehicle to take the responsibility of receiving and resolving the navigation message of NMEA-0183 data format to acquire the information such as longitude, latitude, vehicle speed and direction. The data is then transmitted to monitoring centre through a wireless communication module. The location of vehicle can be clearly monitored on the electronic map at the monitoring centre.

GPS location pinpointing is highly dependent on the electronic map but its accuracy is doubtable if the map is outdated or inaccurate. Other serious challenges of GPS are the problems of non-line-of-sight (NLOS) of GPS signals and multipath effect. NLOS unable the identification of location while signal multipath effect unable accurate positioning. These lead to the researches on network-based positioning, location prediction and hybrid positioning system.

Vehicle monitoring system with GPS is suitable for tracking purposes. The tracking process will aware the driver to drive carefully and hence realizing the purpose of accident prevention. If accident occurs, immediate action can be taken to save the driver. However, it is less efficient for accident prevention measurement as it is only able to locate the location of the vehicle.

Lin et al developed a real-time remote onboard-diagnostic (OBD) system to detect engine operation system for airpollution monitoring [15]. This system can be considered as a vehicle monitoring system for accident prevention. Engine operation condition is closely related to driving safety especially for heavy vehicles or vehicles that are driven for miles. Poor condition of engine operation system can cause accidents to happen and thus the condition has to be discovered in time to get maintenance.

The amalgamation of GPS, GPRS and GIS technology forms $\mathrm{G}^{3}$ system to perform real-time mobile data surveillance with observation on location and condition of engine operation system [15]. $\mathrm{G}^{3}$ system indeed is a superior onboard monitoring system but it can be better if further improved to monitor other aspects such as condition of driver.

Despite location and condition operation system, drowsy driver is an issue to be concerned in the effort to prevent road accidents. This is because the increasing number of traffic accidents is due to the diminished vigilance level of driver [27]. For long haul/distance drivers, fatigue and drowsiness are hardly avoided. Thus, research works are carried out to investigate approaches to monitor driver condition.

According to [28], physiological measures such as brain waves, heart rate, pulse rate and respiration are the most accurate techniques to determine the level of vigilance of driver. However, it is intrusive because some electrodes have to be attached on the driver and this might cause annoyance to the driver. This is the reason of active research works of integrating computer vision techniques in driver monitoring system as it is a nonintrusive approach.

In [27], a real-time online prototype driver-fatigue monitor has been developed by using remotely located charge-coupleddevice (CCD) cameras equipped with active infrared illuminators to acquire video images of the driver. The visual cues obtained such as eyelid movement, gaze movement, head movement and facial expression will be extracted and 
systematically combined to determine the fatigue level of the driver. This system was validated under real-life fatigue condition with human subjects of different ethnic backgrounds, genders, ages, and under different illumination conditions.

Another real-time monitoring system that has been developed is driver vigilance monitoring system. This system has a nonintrusive prototype computer vision system for monitoring a driver's vigilance in real time [28]. Through the visual behavior observed from the captured images, the software can characterize the level of vigilance of the driver. The six significant parameters are Percent Eye Closure (PERCLOS), eye closure duration, blink frequency, nodding frequency, face position, and fixed gaze. These parameters are combined using fuzzy classifier to infer the level of inattentiveness of the driver.

The researchers realized the inconvenience of intrusive devices and thus utilized nonintrusive camera to capture consecutive images [27]-[30]. These vision-based systems require image processing technique to analysis the images Bayesian Network (BN) model [27] and Fuzzy Logic [28]-[30] can be used to deduce the level of drowsiness of driver. The warning system will be triggered once the driver is detected in drowsy condition.

The drowsiness warning system is illustrated as shown in Figure 2. This system is in-vehicle warning system that could alert the drowsy driver in real-time but the condition could not be monitored by third party. Without real-time monitoring, accident could be hardly prevented and immediate action could not be taken by relevant authorities such as police and hospital. The warning system if damaged could not help in reconstructing of the incident to find the root cause of the accident.

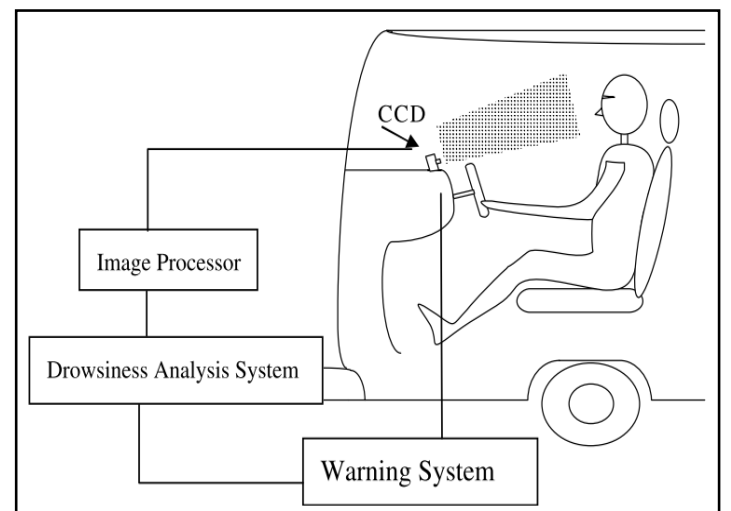

Figure 2 Illustration of drowsiness warning system [30]

On the other hand, alertness of driver can be characterized through the driving performance like lateral position, steering wheel direction and speed [28]. Japanese car maker developed a drowsiness detection system to alert the driver before the vehicle literally drifts out completely [31]. A camera is installed at the front of vehicle to examine the white lines on the road ahead and determine how fast the car is veering towards or away from the detected white lines. The onboard microcontroller will determine the performance of driver according to data collected and computed by an algorithm. The alarm system will be triggered if the driver is judged to be losing consciousness.

Lane departure warning system [32] with concept similar to [31] adopts cameras to acquire images of driving situations and lane geometries. With the images, vehicle dynamic information and the driver's main active maneuver input gathered, the driving scenarios as shown in Figure 3 can be illustrated. In this example, only case 1 activates the warning alarm as the vehicle drifting towards the lane boundary without showing intention to make a lane change. The drifting may due to inattention or drowsiness of driver and the alarm will alert the driver to avoid any potential collision.

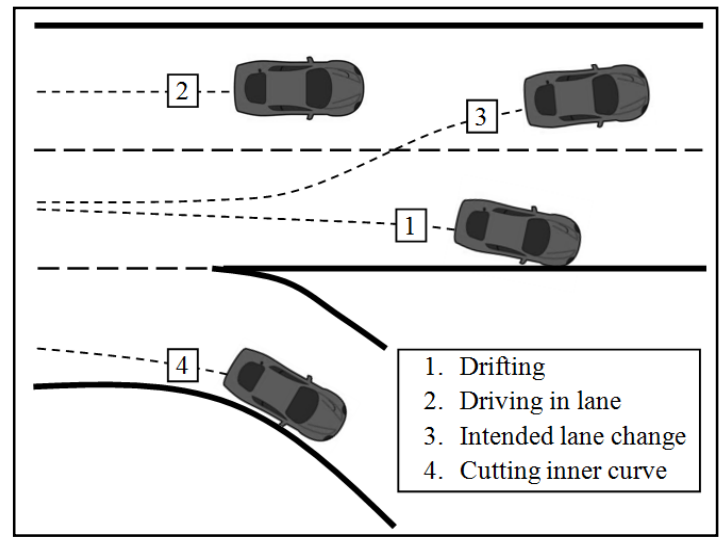

Figure 3 Example of typical driving situations [32] 
Although vision-based technique is nonintrusive to gather images of driver and surroundings, it is sensitive to environmental conditions such as harsh weather. Subsequently, nonintrusive radar sensor is considered to be used to detect the road boundaries and laser sensor to detect white line markings to assist driver to drive in lane.

Speeding is a common factor of road accidents. Driver might be speeding unintentionally or intentionally. Unintentional speeding may be due to unawareness of current speed or habitual behavior while intentional speeding may be due to peer pressure, releasing tension or time saving [33]. A lot of efforts have been taken by the government to help reducing the number of accidents. Government tries to make people aware about the danger of speeding through awareness campaign and limits the driving speed, as well as the construction of street bumps at critical accident areas.

Since the efforts of awareness campaign and enforcement are seem to be less effective, Intelligent Speed Adaptation (ISA) as a kind of Advanced Driver Assistance System (ADAS) is investigated and implemented for safety purpose in some countries. There are four types of ISA systems, which are information, advisory, supportive and intervening systems. Information system informs the driver about the current speed limit; advisory system issues visual, auditory or tactile warning if the speed limit is exceeded; supportive system exerts a counterforce at acceleration pedals if the vehicle is at the speed threshold; intervening system makes it impossible for the vehicle to exceed speed limit.

The results of ISA implementation is promising [34] and is expected to reduce the number of speed violations and thus reduce the number of accidents. However, the objective can only be achieved if ISA large-scale implementation becomes reality with public acceptance because drivers have to agree to install the device on their vehicles. Information and advisory systems are easier to be accepted by public but supportive and intervening systems are mostly refused to be installed. Moreover, the speed violation is unable to be monitored and recorded by third party as evidence.

On the other hand, another serious factor of accident is drunk driving. Thus, drunk driving and rash driving prevention systems have been developed [35]. These systems are designed with an infrared breath analyzer which is placed at the steering wheel to detect the alcohol content in driver's breathe. The infrared absorption is higher when the concentration of ethanol is higher.

Once the alcohol level is detected exceeding certain limit, the alarm is automatically triggered to alert the authorities and the passer by. Thus, the driver is forced to bring the vehicle to stop. In the case of repeat offenders who are more prone to drunk driving, more stringent alternative of cutting fuel supply may be used. A Short Message Service (SMS) with current GPS location of the vehicle is sent to police control room to alert the police. The system is foolproof and the driver cannot tamper with it easily [35].

Nevertheless, research for drunk driving is not as popular as GPS, GIS, GSM, GPRS, image processing, fuzzy logic and data fusion. Therefore, this monitoring system is not implemented even though drunk driving has been a serious issue. Nonintrusive drunk driving monitoring system is suggested to be implemented worldwide for accident prevention.

In addition, the on-road vehicle monitoring system has a fixed monitoring station installed at the roadside or junction with its unique Internet Protocol (IP) address [16]-[17]. At the fixed roadside monitoring station, there are sensors, microcontroller and cameras installed. Sensors detect the violations such as running red light or over-speed vehicle. The outputs of sensors will be the inputs for microcontroller. Then, the microcontroller will process the inputs and define either there is a violation or not while the cameras will take photos of the violation scene.

For the case of running red light, the interrupt procedures will be triggered during the period of red state of traffic light [16]. Once a vehicle passes the first magnetic loop sensor, the interrupt is activated and first image is captured before touching the stabling siding as shown in Figure 4. As the vehicle passes the second sensor, two images of vehicle will be screened as results.

For the case of over-speed, two piezoelectricity sensors are installed on the roadways by a fix distance as shown in Figure 5. The speed can be calculated in real time once the vehicle drives through the two sensors [17]. If the speed exceeds threshold, two images of the vehicle will be screened as result.

The captured first image is used to recognize vehicle plate number while second image is used to record the freeway panorama. Then, the data package consists of violation time, violation place serial number and compressed pictures will be sent to remote monitoring centre [16]-[17].

Meanwhile, vehicle monitoring system developed in [18] set a video frequency monitoring centre at the roadside or junction to gather the scene vehicle image. A camera is used to perform real-time monitoring and transmits the monitoring image to long distance administrative centre after compression. The system uses the dynamic difference background images law to judge the monitoring image. When the movement of vehicle is conforming to the rule, it takes a snapshot and transmits the images to car license recognition software module. The distinguished car-license information will be sent to the 
administrative centre. When exception occurs, the alarm system will be activated immediately. This stable and reliable system is widely applied in vehicles' management [18].

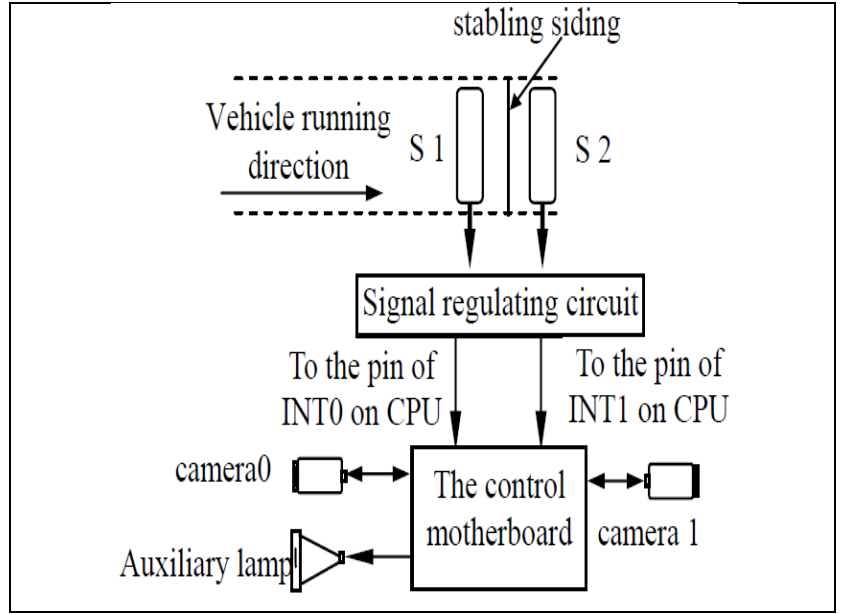

Figure 4 The structural chart of the monitoring station for running red led [16]

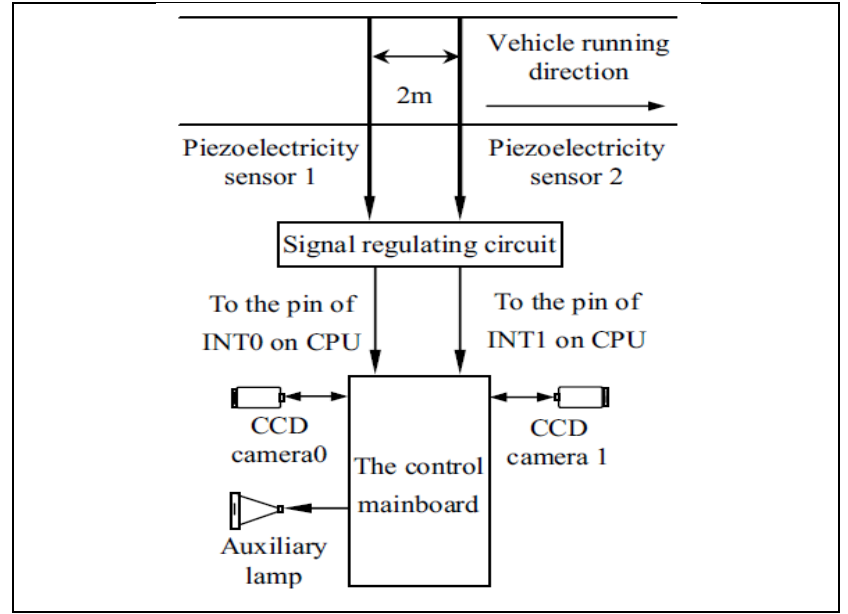

Figure 5 The structural chart of vehicle over-speed monitoring station [17]

\section{B. Wireless Communication Link}

Wireless communication link plays a significant role in completing the two-way communication between vehicle terminal and monitoring centre. Among the various kind of wireless communication technologies, GSM and GPRS are the most extensively used technologies in the vehicle monitoring system due to wide area coverage and high reliability. GSM technology is deployed in [9], [19]-[22] while GPRS technology is deployed in [15]-[18].

According to the established protocols, the information of vehicle location is sent to GSM network in the form of short message by GSM module. The GSM network transfers the received information to the communication gateway of the monitoring centre. The communication between vehicle terminal and monitoring centre is illustrated in Figure 6. The communication connecting communication servers and GSM network adopts the Digital Data Network (DDN) special line or the wireless modem depending to their availability [9]. If the number of vehicles to be monitored is large and the communication condition permits, the DDN special line can be adopted to perform rapid communication with less congestion.

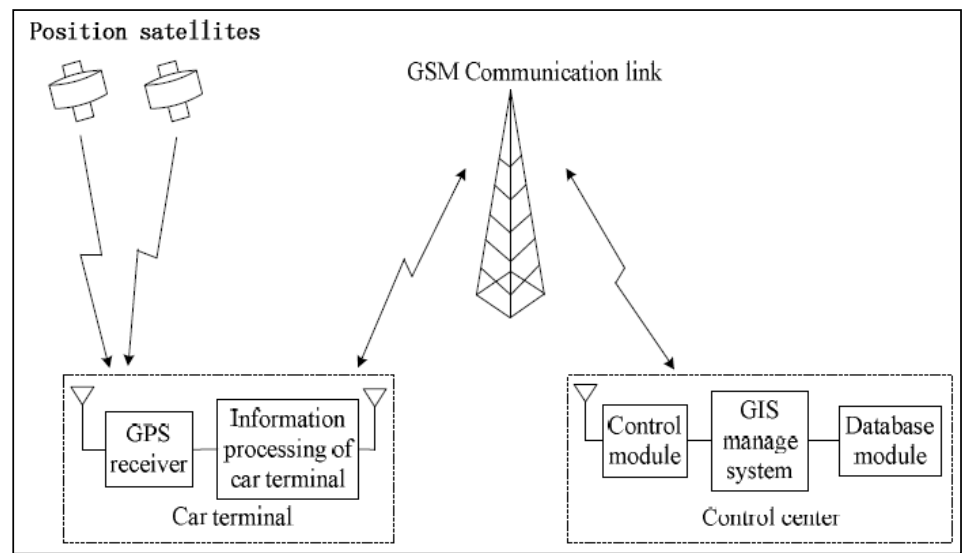

Figure 6 GSM communication link [21]

On the other hand, the vehicle monitoring system which is based on GPRS establishes the communication between vehicle terminal and monitoring system by building up a virtual link between GPRS and internet network. The communication is realized through IP packet. So, the data transmission mode should obey the requirement of GPRS transfer protocol. GPRS data transmission process is done by adding IP header and trailer to the information data by following User Datagram Protocol (UDP) and then encapsulating into IP data packets.

Due to the data traffic between two different modules, Point-to-Point Protocol (PPP) needs to be used to issue the information from micro-processing computer to GPRS wireless data communication module through serial port [11]. The IP data packets are encapsulated into PPP frame before sent to GPRS wireless transmission module.

The wireless transmission module should convert the PPP frame message into SMS message, delivering to Service GPRS Support Node (SGSN). Then, the GPRS Tunnel Protocol (GTP) is encapsulated into package to send to appropriate Gateway 
GPRS Support Node (GGSN) by GPRS backbone networks for making the appropriate protocol transition, as shown in Figure 7. Finally, the data which is encapsulated into new packet according to its external data network protocol will be sent to monitoring centre with specific IP address.

In GPRS transfer mode, the vehicle terminal sending information to monitoring centre is called push up while the monitoring centre sends information to vehicle terminal is called push down [11]. The information format is in compliance with the provisions of communication protocol format defined requirements. For example, if the correct information is received, a confirm code is returned. Meanwhile, if the error information is received, an error code is returned.

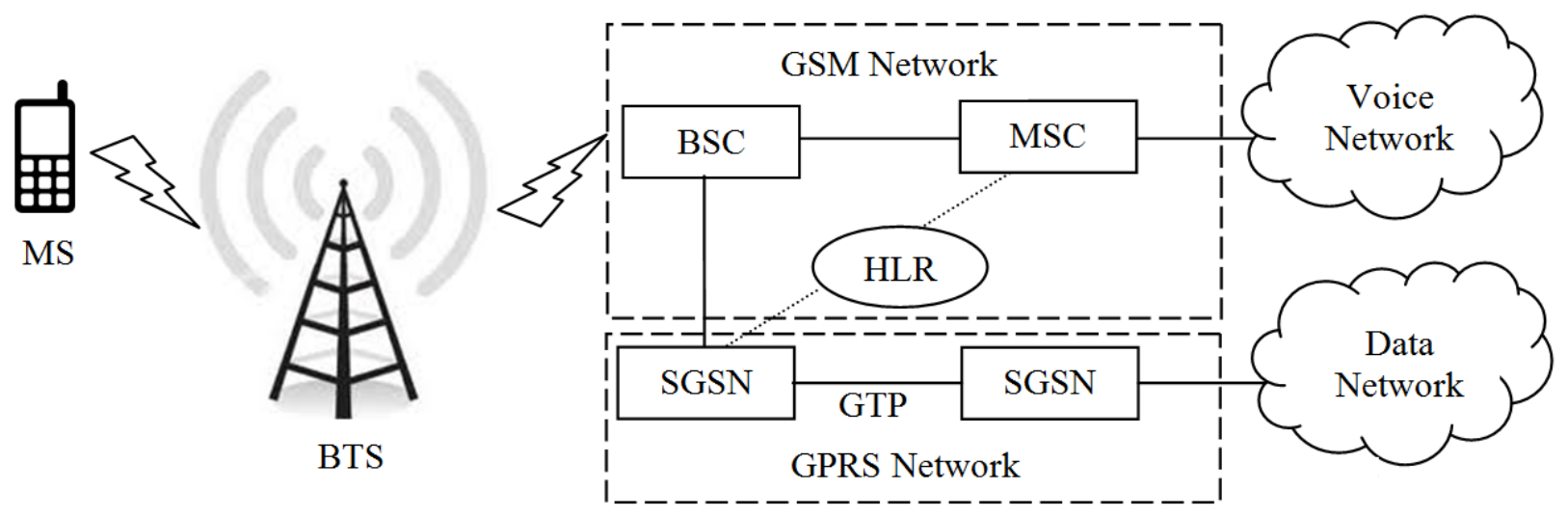

Figure 7 GPRS architecture network

Although there are existing GSM and GPRS infrastructures in most urban areas, there are places without GSM and GPRS signal coverage, giving rise to the challenge of data transmission. In spite of acceptable transmission speed and also reliability, the data charge rate is questionable. If the subscriber is charged over a high volume of data transmitted, the payment will be a financial burden to the user.

Since the GSM network is charging the users according to the number of SMS, the charge rate will be higher compared to GPRS which charges over the volume of data. In fact, there is no wireless communication link provider provides services without charges. The only solution to this issue is by finding a service provider with the lowest charge rate or requires subsidies from government if the system is to be strictly implemented in the country.

\section{Monitoring Centre}

Monitoring centre is used to judge the situation over the time at a remote area. GIS is adopted in most of the vehicle monitoring systems as the geographical information is the most vital element to locate the vehicle [19]-[21]. The monitoring centre mainly process, display and manage the vehicle position and alarm information received.

The main functions of monitoring centre is to track, clear, and refresh the monitored vehicle on electronic map as well as display the detailed database information such as license plate number, vehicle type, driver, contact number and so forth. The system processes alarm information automatically if accident occurs.

In case of emergencies such as robberies or thefts, the monitoring personnel can issue stop instruction to the monitored vehicle by pressing a button. Besides, the system has common map tools for zoom-in and zoom-out. Last but not least, the monitoring centre can perform historical track or data playback with the existence of database module [19]-[21].

With the rapid development of information technology, internet is highly utilized in vehicle monitoring system [19]-[20]. At the monitoring centre, internet is the important connection protocol between client and server. Furthermore, GIS system based on internet provides functions such as map display, vehicle position display, and address query [19].The web system is also based on internet to provide functions such as unit query, vehicle status query, and driver status query as well as relevant data maintenance.

The sequence diagram for connection between client and server is shown in Figure 8. When the client sends connection request to the server, the client initiates the connection thread. Connection thread is responsible for establishing the connection between client and server [20]. Besides, it checks whether there is network fault or breakdown. It reconnects to the server if the connection is break.

The server will read the configuration file before decide the validation of client. If the client is valid, the server will accept the connection request. Then, the connection is established. Next, server sends data file to client while client receives the data when the given port has data. At the same time, the client reads UDP configuration file saving the secondary clients IP address and port. 
The client establishes a socket for secondary clients based on UDP protocol. The client will send data to all secondary clients in the local area network. The secondary clients receive binary data, parse into fixed data structure, save in database, and display the vehicle running state in GIS software.

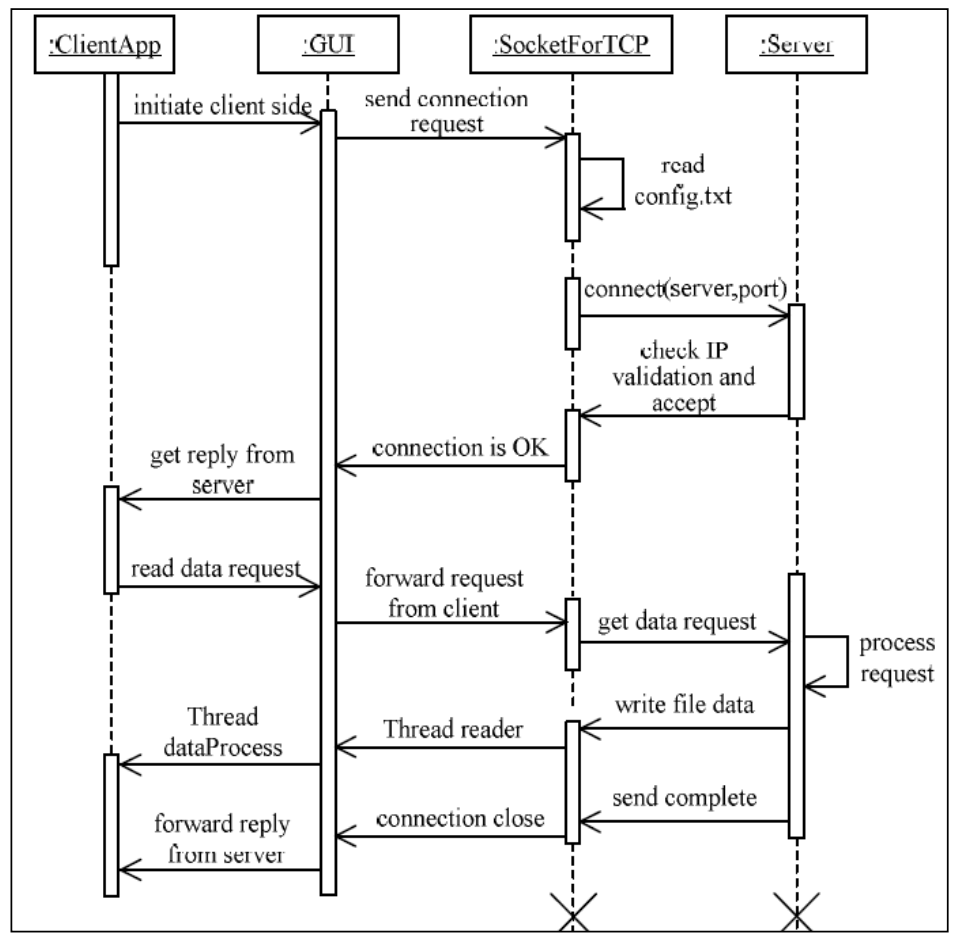

Figure 8 Sequence diagram between client and server [20]

MapX, which is an OCX component developed by MapInfo Corporation, can be quickly integrated into client side application using object-oriented programming languages such as Visual Basic, Visual C++, PowerBuilder or Delphi [20], [22]. MapX offers the easiest and the most cost-effective way to embed mapping functionality into new and existing application without acquiring online mode. MapX can solve the two aspects of map module, which are displaying electronic map and tool setting of map operating [21].

With the electronic map, information can be displayed in a format which is easier to understand. Moreover, map is more informative than charts and graphs. Figure 9 shows the example of Graphical User Interface (GUI) which displays the vehicle information in both graphic format and data grid format.

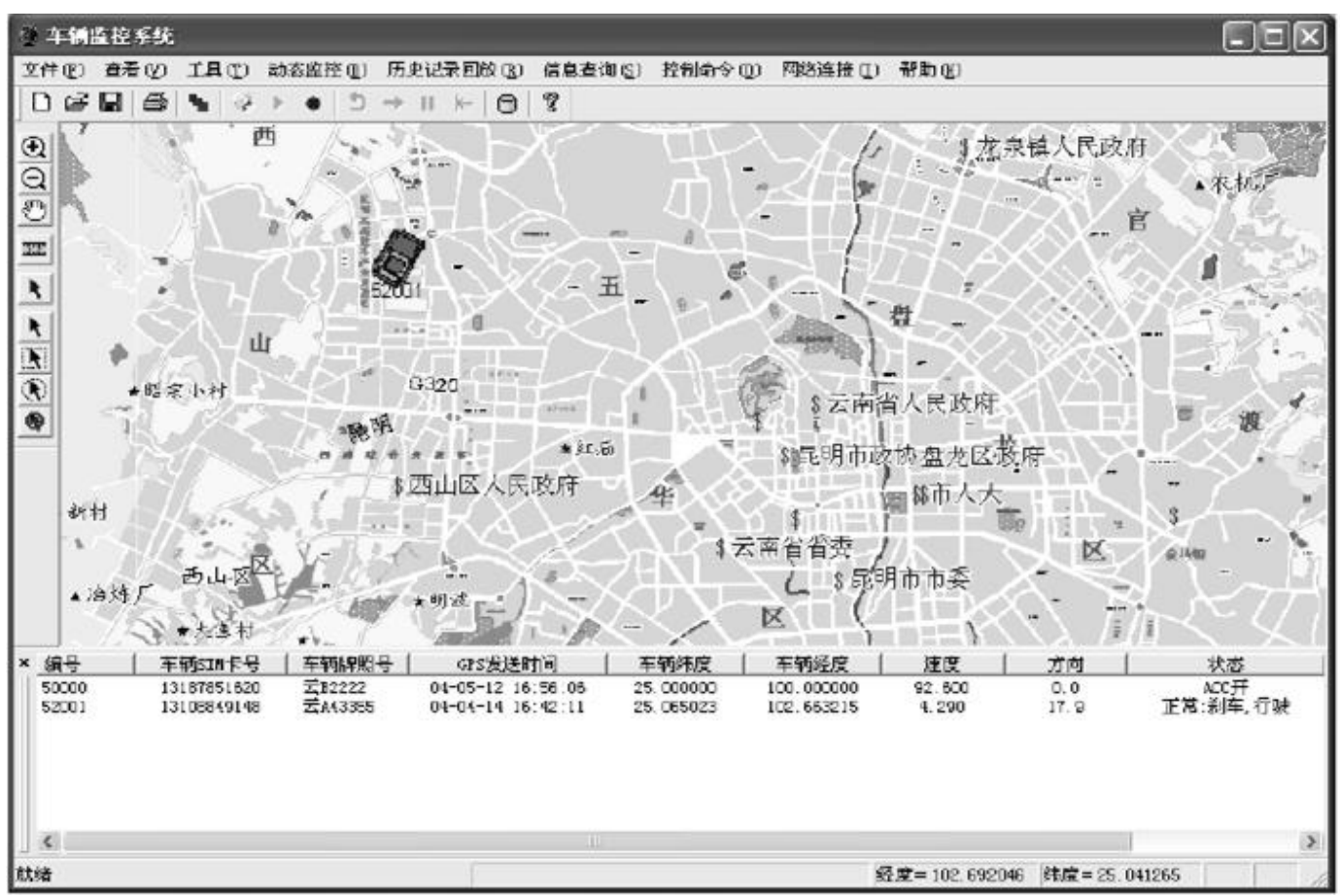

Figure 9 Graphical user interface [20] 
The map data is extremely large and a reliable supportive database is required. Besides, the application must be supportive to handle the large amount of input data. System that is incompatible could not perform smoothly and the lagging will cause the real-time monitoring process to fail.

\section{ISSUES AND CHALLENGES}

Each kind of technology, technique, approach or system has its pros and cons with additional issues and challenges. Vehicle monitoring system for accident prevention is a system that requires high mobility and wide coverage is believed to have its issues and challenges especially in its accuracy, integrity, security, cost and public acceptance.

The accuracy and precision of the surveillance system is the main issue in vehicle monitoring system. For positioning, GPS is the most common technology. However, it has the drawback of NLOS and multipath effect that may degrade its performance at certain area such as forest, tunnel, and rural area. Thus, research on integration of GPS with other positioning technologies like satellite-based augmentation system, network-based positioning and location prediction is necessary to increase the accuracy and precision of positioning.

Other than positioning, surveillance system in vehicle highly depends on the input data from range of sensors to observe the condition of vehicle and driver. Thus, the accuracy of variety sensors should be verified before implemented in vehicle monitoring system to assure the detected information is accurate and precise. Some of the surveillance system deploys the use of camera. Therefore, the performance of image processing technology affects the accuracy of the output. The deviation of information may cause false alarm which leads to inconvenience to users.

Since the vehicle monitoring system requires input from various input sources, the integrity of information becomes main challenge. Different kind of sensors has heterogeneous nature of information that needs to be integrated. Hence, different data fusion approaches need to be adopted for the integrity of the applications. Although data fusion approaches including statistical approach, probabilistic approach and artificial intelligence are investigated and implemented in some applications, the high load of real-time data is burdening the system. High performance processor with appropriate data fusion approach is required in realizing the integrity of system.

Apart from that, the privacy and security are also issues in vehicle monitoring system. Since the system detects the location of the vehicle, condition of the vehicle as well as the driver and transmits it to the monitoring centre, public concern on privacy and security issue is common. The system is at a risk to be hacked. Once the details of a person are exposed, the data may be misused to involve in criminal activities. Consequently, data protection must be enhanced to its maximum before implementation.

A comprehensive vehicle monitoring system is normally costly due to its complexity and intelligence. Moreover, system that requires wireless communication system needs to be charged for the data transmission according to the up to date data rate. The financial burden discourages the installment of such safety system. Therefore, a low cost vehicle monitoring system with compatible performance along with lowest data charge rate should be developed.

Based on the issues and challenges mentioned, it is difficult for the public to accept such system. Although it is nonintrusive, it may be rejected by most of the people as it requires extra installation with extra payment and the risk to be tracked by unethical person. The best solution to this public acceptance problem is by force implementation with subsidies for the installation and data charge.

\section{CONCLUSION}

The recent vehicle monitoring systems are developed by using GPS technology. In the case of NLOS, the satellite-based positioning technology is invalid to locate the vehicle. In addition, most of the vehicle monitoring systems emphasize on the location of vehicles but not the condition of vehicles and drivers. Meanwhile, the monitoring system which can monitor the condition of drivers is not integrated with the real-time positioning technologies. The monitoring system with fixed work station is useful in monitoring scenarios such as running red light and over-speed for accident prevention but this kind of monitoring system is installed at certain places only. For the wireless communication link, GSM and GPRS are stable and reliable technologies with added value that no infrastructure installation is needed but the data charge is questionable. With respect to the monitoring centre, the systems are too dependent on internet access which should be avoided due to the fact that some remote monitoring centres are located in rural areas with poor network coverage. MapX is considered as one of the best GIS mapping technique as it does not acquire online mode.

\section{ACKNOWLEDGMENT}

The author would like to thank Universiti Malaysia Sarawak (UNIMAS) for the research fund under the Dana Principal Investigator (DPI), Grant No.: Dana Principal Investigator (DPI)-02 (DPI15)873/202(07). 


\section{REFERENCES}

[1]. Z. Wei, P. Zhao, and S. Ai, "Efficiency Evaluation of Beijing Intelligent Traffic Management System Based on Super-DEA," in Journal of Transportation Systems Engineering and Information Technology, vol. 12, no. 3, pp. 19-23, Jun. 2012.

[2]. S. C. Davis, S. W. Diegel, and R. G. Boundy, Transportation Energy Data Book: Edition 31, 31st ed. United States: Center for Transportation Analysis Energy and Transportation Science Division, 2012.

[3]. J. He, Z. Zeng, and Z. Li, "Benefit Evaluation Framework of Intelligent Transportation Systems," in Journal of Transportation Systems Engineering and Information Technology, vol. 10, no. 1, pp. 81-87, Feb. 2010.

[4]. MIROS. (2011). General Road Accident Data in Malaysia (1995 - 2010). [Online]. Available: http://www.miros.gov.my/web/guest/road

[5]. M. Irfan, M.M. T. N. Baig, R. M. Hashmi, F. H. Khan, K.Shehzad and A. Ali, "Management of Location Based Advertisement Services using Spatial Triggers in Cellular Networks," in International Journal of Computer Science and Information Security (IJCSIS),vol. 6, no. 1, pp. $181-185$, Jan. 2009.

[6]. S. Cojocaru, E. Barson, G. Batrinca and P. Arsenie, "GPS-GLONASS-GALILEO: A Dynamical Comparison," in Journal of Navigation, vol. 62, no. 1, pp. 135-180, Jan. 2009.

[7]. C. J. Hegarty and E. Chatre, "Evolution of the Global Navigation Satellite System (GNSS)," in Proceedings of the IEEE, vol. 96, no. 12, pp. 19021917, Dec. 2008

[8]. C. F. Prades, L. L. Presti, and E. Falletti, "Satellite Radiolocalization from GPS to GNSS and Beyond: Novel Technologies and Applications for Civil Mass Market," Proceedings of the IEEE, vol. 99, no. 11, pp. 1882-1904, Nov. 2011.

[9]. Q. Liu, H. P. Lu, H. L. Zhuang and B. Zou, "Research and Design of Intelligent Vehicle Monitoring System Based on GPS/GSM," in 2006 6th International Conference on ITS Telecommunications Proceedings, pp. 1267-1270, Jun. 2006.

[10]. J. H. Xie and J. P. Zhong, "Design and Implementation of GPS Vehicle Monitoring Information System," in International Conference on Information Engineering and Computer Science (ICIECS) 2009, pp. 1-4, Dec. 2009.

[11]. East-West Digital News. (2011, June 02). Glonass Sets Goal to Exceed GPS Accuracy Twofold; Vice PM Guarantees Free Service Worldwide. [Online]. Available: http://www.ewdn.com/2011/06/02/.

[12]. N. E. E. Faouzi, H. Leung, and A. Kurian, "Data Fusion in Intelligent Transportation Systems: Progress and Challenges - A Survey," in Information Fusion, vol. 12, no. 1, pp. 4-10, Jan. 2011.

[13]. X. Chen, X. Jiang, and L. Wang, "Development on ARM9 System-on-chip Embedded Sensor Node for Urban Intelligent Transportation System," in 2006 IEEE International Symposium on Industrial Electronics, pp. 3270-3275, Jul. 2006.

[14]. M. L. Sichitiu and M. Kihl, "Inter-Vehicle Communication Systems: A Survey," in IEEE on Communication Surveys and Tutorials, vol. 10, no.2, pp. 88-105, Jul. 2008.

[15]. C. E. Lin, Y. S. Shiao, C. L. Li, S. H. Yang, S. H. Lin, and C. Y. Lin, "Real-time Remote Onboard Diagnostics using Embedded GPRS Surveillance Technology," Vehicular Technology, vol. 56, no. 3, pp. 1108-1118, May. 2007.

[16]. Z. Q. Ma, D. Y. Chen and L. Cui, "Wireless Monitoring System of Vehicle Violation of Running Red Led Based on GPRS," in 3rd International Conference on Innovative Computing Information and Control (ICICIC) 2008, pp. 240-240, Jun. 2008.

[17]. Z. Q. Ma, G. S. Gao, W. M. Song and Y. Yan, "Wireless Monitoring System of Vehicle Overspeed on Freeway Based on GPRS," in 2008 27th Chinese Control Conference on CORD Conference Proceedings, pp. 550-553, Jul. 2008.

[18]. H. M. Yu, Z. F. Pang and D. R. Ruan, "The Design of the Embedded Wireless Vehicles Monitoring Management System Based on GPRS: Evidence from China," in International Symposium on Intelligent Information Technology Application Workshops (IITAW) 2008, pp. 677-680, Dec. 2008.

[19]. H. Tan, "Design and Implementation of Vehicle Monitoring System Based on GSM/GIS/GPS," in 2010 Second International Conference on Information Technology and Computer Science (ITCS), pp. 413-416, Jul. 2010.

[20]. P. Chen and S. Liu, "Intelligent Vehicle Monitoring System Based on GPS, GSM and GIS," in 2010 WASE International Conference on Information Engineering (ICIE), pp. 38-40, Aug. 2010.

[21]. H. Hu and L. Fang, "Design and Implementation of Vehicle Monitoring System Based on GPS/GSM/GIS," in Third International Symposium on Intelligent Information Technology Application (IITA)2009, pp. 278-281, Nov. 2009.

[22]. D. Liu, Z. Liang, and Y. Li, "Research and Design of A High performance GPS Vehicle Monitoring System," in 2010 International Conference on Future Information Technology and Management Engineering, pp. 59-62, Oct. 2010.

[23]. Y. Sugawara, "Understanding the Differences in the Development and Use of Advanced Traveler Information Systems for Vehicles (ATIS/V) in the U.S., Germany, and Japan,” Massachusetts Institute of Technology, Jun. 2007.

[24]. J. Song, N. N. Li, J. L. Chen, Y. F. Dong, and Z. Zhao, "Design and Implementation of Intelligent Transportation System Based on GPRS and Bluetooth Hybrid Model," in Proceedings of the IEEE International Conference on Automation and Logistics, no. September, pp. 1381-1385, Sep. 2008.

[25]. A. M. Mustapha, M. Hannan, A. Hussain, and H. Basri, "UKM Campus Bus Monitoring System using RFID and GIS," in 2010 6th International Colloquium on Signal Processing \& its Applications (CSPA), pp. 62-66, May. 2010.

[26]. P. Fu and J. L. Sun. Web GIS: Principles and Applications. New York Street, Redlands, California: ESRI Press, 2011.

[27]. Q. Ji and Z. Zhu, "Real-time Nonintrusive Monitoring and Prediction of Driver Fatigue," in Vehicular Technology, IEEE Transactions, vol. 53, no. 4, pp. 1052-1068, Jul. 2004.

[28]. L. Bergasa, J. Nuevo, and M. Sotelo, "Real-Time System for Monitoring Driver Vigilance," in IEEE International Symposium on Industrial Electronics, pp. 1303-1308, Jun. 2005.

[29]. N. Sharma and V. K. Banga, "Development of a Drowsiness Warning System Based on The Fuzzy Logic," in International Journal of Computer Applications, vol. 8, no. 9, pp. 1-6, Oct. 2010.

[30]. J. Wu and T. Chen, "Development of a Drowsiness Warning System Based on The Fuzzy Logic Images Analysis," in Expert Systems with Applications, vol. 34, no. 2, pp. 1556-1561, Feb. 2008.

[31]. M. Justin. (2009, Jan 23). Invention: Sleepy Driver Sensor. [Online]. Available: http://www.newscientist.com/article/dn16478-invention-sleepy-driversensor-.html.

[32]. A. Kummert, S. B. Park, and D. Neisius, “A Warning Algorithm for Lane Departure Warning System,” in 2009 IEEE Intelligent Vehicles Symposium, pp. 431-435, Jun. 2009.

[33]. K. L. Young, M. a Regan, T. J. Triggs, K. Jontof-Hutter, and S. Newstead, "Intelligent Speed Adaptation-Effects and Acceptance by Young Inexperienced Drivers," in Accident Analysis and Prevention, vol. 42, no. 3, pp. 935-43, May 2010.

[34]. H. W. Warner, T. Özkan, and T. Lajunen, "Drivers' Propensity to have Different Types of Intelligent Speed Adaptation Installed in Their Cars," in Transportation Research Part F: Traffic Psychology and Behaviour, vol. 13, no. 3, pp. 206-214, May 2010.

[35]. T. S. Ramanath, A. Sudharsan and U. F. Udhayaraj, "Drunken Driving and Rash Driving Prevention System," in 20102 nd International Conference on Mechanical and Electrical Technology (ICMET), pp. 599-603, Sep. 2010. 